\title{
Obtenção de Consentimento Informado para Anestesia em Cirurgia Eletiva num Hospital Terciário: Práticas e Contexto Ético-Legal
}

\section{Obtaining Informed Consent for Anesthesia in Elective Surgery at a Tertiary-Care Hospital: Practices and Ethical- Legal Context}

\author{
Luís Guilherme CASIMIRO $\rrbracket^{1}$, Sara PEREIRA², Sofia PIRES ${ }^{3}$, Joana MOURÃO ${ }^{1,2}$ \\ Acta Med Port 2019 Jan;32(1):53-60 - https://doi.org/10.20344/amp.10592
}

\section{RESUMO}

Introdução: O consentimento informado é um processo ativo na relação médico-doente, assente em valores éticos e legais. O ato anestésico tem riscos inerentes, que devem ser alvo de consentimento específico. O objetivo deste estudo foi avaliar o grau de implementação do consentimento informado escrito para o ato anestésico no contexto de cirurgia eletiva.

Material e Métodos: Estudo observacional prospetivo, num hospital terciário e universitário, em 230 doentes com idade igual ou superior a 60 anos submetidos a cirurgia eletiva entre maio e julho de 2017. Aos doentes elegíveis que consentiram participar, foi realizada entrevista clínica no dia prévio à cirurgia. No pós-operatório, foi averiguada a técnica anestésica realizada, e a existência do consentimento informado por escrito para o ato anestésico e cirúrgico. Doentes incapazes de dar consentimento informado ou admitidos na unidade de cuidados intensivos após cirurgia foram excluídos.

Resultados: Em 225 (97,8\%) dos doentes, verificou-se a obtenção, por escrito, do consentimento informado para o ato cirúrgico, mas apenas em $96(41,7 \%)$ verificou-se a obtenção por escrito do consentimento informado para o ato anestésico. De entre os doentes sem registo de consentimento informado para o ato anestésico, foram mais prevalentes antecedentes de acidente vascular cerebral, anemia e scores de Charlson e de estado físico conforme à Sociedade Americana de Anestesiologia mais elevados.

Discussão: Identificámos uma baixa implementação do consentimento informado escrito para o ato anestésico. Esta situação pode ter importantes implicações em contexto de responsabilidade disciplinar, civil ou penal.

Conclusão: Apesar da sua importância, a prática do consentimento informado escrito para o ato anestésico nesta instituição não está implementada regularmente.

Palavras-chave: Anestesia; Consentimento Informado/ética; Consentimento Informado/legislação e jurisprudência; Procedimentos Cirúrgicos Eletivos; Responsabilidade Legal; Termos de Consentimento

\section{ABSTRACT}

Introduction: Informed consent is an active process of the doctor-patient relationship, based on ethical and legal principles. The anesthetic act has inherent risks, which should be subject of specific consent. The aim of this study was to evaluate the degree of implementation of written specific informed consent for anesthesia in the context of elective surgery.

Material and Methods: An observational prospective study, at a tertiary university hospital, in 230 patients aged 60 years or older undergoing elective surgery between May and July 2017. Eligible patients who consented to participate were interviewed clinically on the day before surgery. In the postoperative period, the anesthetic technique and the existence of the written informed consent for the anesthetic and surgical procedures were assessed. Patients who were unable to give informed consent or those admitted in the Intensive Care Unit after surgery were excluded.

Results: Written informed consent for the surgical procedure was obtained for 225 (97.8\%), while it was obtained in just 96 (41.7\%) patients for the anesthetic act. There was a higher prevalence of stroke, anemia, and higher Charlson and physical American Society of Anesthesiologists scores in patients without written informed consent for the anesthetic act.

Discussion: We identified a low implementation of written informed consent for anesthesia. This situation may have important implications in the context of disciplinary, civil or criminal liability.

Conclusion: Despite its importance, the practice of written informed consent for anesthesia in this institution is not yet implemented on a regular basis.

Keywords: Anesthesia; Consent Forms; Elective Surgical Procedures; Informed Consent/ethics; Informed Consent/legislation \& jurisprudence; Liability, Legal

\section{INTRODUÇÃO}

A obtenção do consentimento informado $(\mathrm{Cl})$ pressupõe o esclarecimento do doente acerca da proposta terapêutica, médica ou cirúrgica, e, com base no seu sistema de valores, este decide aceitá-la ou não. ${ }^{1}$ Para ser válido e cumprir o seu propósito, deve constituir um processo ativo

de diálogo participado nas decisões sobre saúde do doente. ${ }^{2} \mathrm{O}$ doente deve ser esclarecido sobre todos os riscos inerentes ao procedimento de forma clara e objetiva, sem discrepância de informação face ao profissional de saúde. A decisão do doente, deve ser reconhecida como uma

1. Serviço de Anestesiologia. Centro Hospitalar São João. Porto. Portugal.

2. Departamento de Anestesiologia. Faculdade de Medicina. Universidade do Porto. Porto. Portugal.

3. Departamento de Investigação e Ação Penal. Ministério Público. Porto. Portugal.

$\square$ Autor correspondente: Luís Guilherme Casimiro. luisg.casimiro@gmail.com

Recebido: 01 de abril de 2018 - Aceite: 24 de setembro de 2018 | Copyright @ Ordem dos Médicos 2019 
manifestação legítima de autonomia, desde que livre e esclarecida. $^{3}$

$\mathrm{O} \mathrm{Cl}$ é valorizado sob dois pontos de vista: o ético, como peça fundamental da relação médico-doente e da autonomia do doente; o legal, enquanto instituto que permite a autodeterminação sobre os riscos assumidos, delimitando a responsabilidade que recai sobre o médico. ${ }^{4} \mathrm{O} \mathrm{Cl}$, particularmente se registado por escrito, constitui um instrumento documental e prova de defesa num eventual processo judicial de responsabilidade médica. Está consagrado em várias normas legais e deontológicas, como a Convenção de Oviedo, ${ }^{5}$ a Lei de Bases da Saúde, ${ }^{6}$ o Estatuto ${ }^{7}$ e Código Deontológico da Ordem dos Médicos, ${ }^{8}$ a Norma 015/2013 da Direção-Geral da Saúde, ${ }^{3}$ o Código Civil $^{9}$ e o Código Penal. ${ }^{10}$

O consentimento para atos anestésicos foi durante muito tempo considerado implícito no caso de intervenções cirúrgicas eletivas. $O$ cirurgião dominava quase exclusivamente o bloco operatório, não existindo autonomia ou responsabilidade dos restantes intervenientes, independentemente da sua diferenciação. Assim, ficava a seu cargo apresentar a proposta anestésica ao doente. Gradualmente, a Anestesiologia afirmou-se como especialidade diferenciada, com campos de intervenção próprios e autónoma em múltiplas competências, que superam o ato de anestesiar. A relação entre cirurgião e anestesiologista tornou-se horizontal, e o contacto direto entre o doente e o anestesiologista convoca a sua responsabilidade pessoal e civil em regime cumulativo, vinculando-o à obrigação de meios ${ }^{11}$ e à obtenção de consentimento livre e esclarecido para as técnicas que propõe.

A autonomia do anestesiologista em relação à restante equipa está consagrada no Código Deontológico da Ordem dos Médicos: "o médico, no exercício da sua profissão, é técnica e deontologicamente independente e responsável pelos seus atos". Porém, os trabalhos de Tait et al e Zarnegar et al mostram que os doentes continuam a atribuir maior importância ao consentimento para o ato cirúrgico do que para o ato anestésico, recordando-se melhor da informação discutida acerca da intervenção cirúrgica propriamente dita. ${ }^{12,13}$

$\mathrm{O}$ ato anestésico tem natureza, propósito, benefícios, alternativas e riscos próprios, que devem ser alvo de discussão e consentimento específicos. Apenas o anestesiologista tem as competências e conhecimentos necessários à formulação e discussão de um plano anestésico e suas particularidades. Por esse motivo, a discussão e obtenção do consentimento para o ato anestésico deve ser uma obrigação ética e legal do anestesiologista, e não de outros profissionais de saúde. Vários autores verificaram que a obtenção de $\mathrm{Cl}$ próprio para o ato anestésico melhora a compreensão acerca dos procedimentos anestésicos, seus benefícios e efeitos laterais, assim como do papel do anestesiologista na intervenção. ${ }^{14,15}$

A Direção-Geral de Saúde preconiza as práticas de transmissão de informação adequada e documentação do $\mathrm{Cl}$ sugeridas pela literatura atual. ${ }^{3,16,17}$ Sugere que seja obtido $\mathrm{Cl}$ por escrito, em formulário próprio, para a cirurgia eletiva com recurso a qualquer técnica anestésica. A existência deste formulário constitui uma forma prática de documentar a decisão do doente.

O objetivo deste estudo foi avaliar a obtenção do Cl escrito para o ato anestésico no contexto de procedimentos cirúrgicos eletivos em doentes com idade igual ou superior a 60 anos, num hospital terciário universitário.

\section{MATERIAL E MÉTODOS}

Após aprovação pela comissão de ética institucional, realizámos um estudo observacional prospetivo no serviço de Anestesiologia de um hospital terciário universitário.

$O$ estudo foi desenhado com o intuito de estudar a população idosa submetida a cirurgia eletiva, dada a sua prevalência crescente, as suas comorbilidades, qualidade de vida e outcome cirúrgico. Foram incluídos neste estudo doentes com idade igual ou superior a 60 anos, submetidos a cirurgia eletiva das especialidades de Cirurgia Geral, Urologia, Ginecologia, Cirurgia Plástica, Cirurgia Vascular, Ortopedia ou Cirurgia Maxilo-Facial. Foram selecionados os doentes intervencionados entre maio e julho de 2017. Os doentes admitidos em Unidade de Cuidados Intensivos no pós-operatório, com incapacidade de compreender a língua portuguesa, ou com incapacidade de dar $\mathrm{Cl}$ para participação neste estudo foram excluídos.

Foi realizada uma entrevista clínica no dia prévio à cirurgia onde recolhemos os seguintes dados: procedimento cirúrgico proposto, dados demográficos, antecedentes patológicos, medicação habitual, classificação do estado físico de acordo com a Sociedade Americana de Anestesiologia (ASA), índice de Charlson, escala de fragilidade clínica e capacidade cognitiva avaliada pelo Montreal Cognitive Assessment (MoCA). O índice de Charlson é um indicador com valor prognóstico, que classifica e valoriza as comorbilidades do doente de modo a estimar a sua mortalidade a 10 anos. ${ }^{18,19} \mathrm{~A}$ escala de fragilidade clínica estratifica os doentes de acordo com o seu nível de vulnerabilidade, constituindo um forte preditor de eventos adversos pós-cirúrgicos. ${ }^{20,21}$ O Montreal Cognitive Assessment (MoCA) constitui um método de deteção precoce de disfunção cognitiva, que se encontra adaptado à população portuguesa. ${ }^{22-24}$ Foi considerado um cut-off clínico de $26,{ }^{25}$ abaixo do qual se considerou existir défice cognitivo do doente.

No período pós-operatório, foram obtidos do processo clínico do doente os dados referentes à técnica anestésica e à obtenção de $\mathrm{Cl}$ por escrito para o ato anestésico e para o ato cirúrgico. Apesar de desaconselhado, ainda é frequente o primeiro contacto do anestesiologista com o doente ser no pré-operatório imediato, momento no qual deverá obter o $\mathrm{Cl}$ escrito. Deste modo, a existência do $\mathrm{Cl}$ escrito para o ato anestésico foi apenas averiguada após o procedimento cirúrgico.

Os doentes foram agrupados de acordo com a existência ou não de $\mathrm{Cl}$ por escrito para o ato anestésico, permitindo averiguar a existência, ou não, de condições do doente ou do procedimento anestésico, associadas à ausência de 
Cl por escrito.

Para análise estatística, utilizámos os testes de Mann-Whitney para variáveis contínuas. Para as variáveis categóricas, aplicámos o teste de qui-quadrado de Pearson, o teste de associação linear por linear e o teste exato de Fisher. As diferenças foram consideradas significativas quando $p<0,05$. A análise foi realizada com recurso ao IBM Statistical Package for Social Sciences (SPSS Inc. Chicago, IL), versão 24.0 .

\section{RESULTADOS}

Estudámos 230 doentes, dos quais 124 (53,9\%) eram do sexo feminino e $106(46,1 \%)$ do sexo masculino. A média de idades foi 70,92 anos.

Em 225 doentes (97,8\%), verificámos registo de $\mathrm{Cl}$ por escrito para o ato cirúrgico, enquanto o mesmo foi verificado em apenas $96(41,7 \%)$ para o ato anestésico. Nenhum dos casos de consentimento escrito ausente para o ato anestésico se deveu ao não consentimento por parte do doente.

Em cinco doentes $(2,2 \%)$ verificámos a inexistência de $\mathrm{Cl}$ documentado por escrito, para ambos os procedimentos (cirúrgico e anestésico).

A ausência de $\mathrm{Cl}$ escrito para o ato anestésico associou-se a uma maior prevalência de antecedentes de acidente vascular cerebral $(p=0,021)$ e de anemia $(p=0,028)$, bem como scores de Charlson $^{19}(0,017)$ e classificação de estado físico ASA $(p=0,046)$ mais elevados. Não identificámos associação significativa com as restantes variáveis estudadas, antecedentes de patologia neurológica, psiquiátrica, cardiovascular, ou maior índice de fragilidade. Os resultados obtidos encontram-se discriminados na Tabela 1.

A distribuição dos doentes pelos serviços cirúrgicos encontra-se descrita na Tabela 2. Não foram encontradas diferenças significativas na distribuição dos doentes pelos diferentes serviços cirúrgicos.

\section{DISCUSSÃO}

Apenas 96 (41,7\%) doentes tiveram registo do $\mathrm{Cl}$ por escrito para o ato anestésico, enquanto $225(97,8 \%)$ o tiveram para o ato cirúrgico. Estes resultados são concordantes com trabalhos anteriores, que mostraram uma maior implementação do consentimento para atos cirúrgicos do que para atos anestésicos. ${ }^{12,26} \mathrm{~A}$ ausência de consentimento escrito para o ato anestésico, neste caso, não traduz a ausência de autonomia do doente, uma vez que este consente $o$ ato cirúrgico, mas possivelmente a falta de sensibilização do anestesiologista para esse facto.

De entre o grupo de doentes com ausência de $\mathrm{Cl}$ para o ato anestésico, verificámos uma maior prevalência de doentes com antecedentes de anemia, acidente vascular cerebral, estado físico ASA e Charlson mais elevados. O risco aumentado de complicações particulares, devem ser adequadamente discutidas aquando da obtenção de $\mathrm{Cl}$ para o ato anestésico.

Os doentes com antecedentes de acidente vascular cerebral apresentam a priori territórios vasculares cerebrais debilitados, encontrando-se particularmente suscetíveis às possíveis complicações decorrentes de eventuais episódios intraoperatórios de hipoperfusão cerebral, nomeadamente novo episódio isquémico, e disfunção cognitiva pós-anestésica. ${ }^{27-29}$

A anemia pré-operatória constitui um fator de risco independente de outcomes peri-operatórios adversos ${ }^{30-32}$ e necessidade de transfusão no decurso da intervenção cirúrgica. ${ }^{33,34}$ Estas consequências podem ser potenciadas pela própria anemia iatrogénica decorrente da hemodiluição durante o procedimento, e pela depressão simpática e cardíaca induzida pela anestesia. ${ }^{32,35} \mathrm{~A}$ proposta anestésica deverá ter em conta fatores específicos que podem contribuir pela opção de uma determinada técnica anestésica: a severidade e tipo de anemia, o nível de compensação fisiológica e a perda hemática esperada. A possível indicação para transfusão sanguínea durante o procedimento deverá ser discutida com o doente, respeitando e registando adequadamente a sua vontade.

Os doentes com pontuação mais elevada nos scores de Charlson ${ }^{19}$ e no estado físico ASA apresentam, globalmente, pior condição física e mais comorbilidades, constituindo um grupo mais suscetível à ocorrência de complicações peri-operatórias, necessidade de intervenções não planeadas e sequelas de severidade variável. Como tal, é importante que estes doentes sejam adequadamente informados acerca da sua condição, riscos e alternativas. Esta abordagem deve ser sistematizada no sentido de ajustar a informação às capacidades cognitivas do doente ou, se indicado, informando adequadamente o seu representante legal e obtendo consentimento deste.

Para cinco dos 230 doentes incluídos no estudo $(2,2 \%)$ não foi documentada a obtenção de $\mathrm{Cl}$ por escrito para a intervenção cirúrgica ou anestésica, revelando o incumprimento, por parte da equipa de médicos e enfermeiros do bloco operatório, das indicações para a cirurgia segura segundo a Organização Mundial de Saúde e a Norma 002/2013 da DGS. ${ }^{36,37}$ Esta situação pode criar sérias dificuldades no esclarecimento da licitude das intervenções realizadas, porque apenas o $\mathrm{Cl}$ livre e esclarecido permite transferir para o doente os riscos, de outro modo suportados pelo médico. ${ }^{4}$

Atualmente, o $\mathrm{Cl}$ é um pré-requisito legal em qualquer intervenção médica e fulcral na legitimidade desta, consagrado em várias normas legais e deontológicas, de entre as quais o Código Deontológico da Ordem dos Médicos, ${ }^{8}$ o Código Civil ${ }^{9}$ e o Código Penal. ${ }^{10}$ Permite delimitar a responsabilidade que recai sobre o médico ou sobre o doente ${ }^{38,39}$ pois este, ao consentir, apropria-se conscientemente dos riscos de que foi informado. ${ }^{40} \mathrm{O}$ dever de informar está preconizado a nível disciplinar e jurídico. O anestesiologista tem áreas bem delimitadas de responsabilidade perante o doente, e, portanto, responde direta e pessoalmente por eventual atuação negligente. ${ }^{11}$

Em Portugal, a responsabilidade disciplinar médica é regulada pelo Estatuto Disciplinar Médico ${ }^{41}$ e Código Deontológico da Ordem dos Médicos; ${ }^{8}$ estes instrumentos de 
Tabela 1 - Características demográficas e clínicas dos doentes observados $(n=230)$

\begin{tabular}{|c|c|c|c|c|}
\hline $\begin{array}{l}\text { Variável, } \\
\text { n (\%) ou mediana (P25 - P75) }\end{array}$ & $\begin{array}{c}\text { Total } \\
\mathrm{n}=230\end{array}$ & $\begin{array}{c}\text { Com } \mathrm{Cl} \text { por escrito } \\
\text { para ato anestésico } \\
\mathrm{n}=96(41,7)\end{array}$ & $\begin{array}{c}\text { Sem } \mathbf{C l} \text { por escrito } \\
\text { para ato anestésico } \\
n=134(58,3)\end{array}$ & Valor $p$ \\
\hline $\begin{array}{l}\text { Sexo } \\
\text { Feminino } \\
\text { Masculino }\end{array}$ & $\begin{array}{l}124 \\
106\end{array}$ & $\begin{array}{l}54(43,5) \\
42(39,6)\end{array}$ & $\begin{array}{l}70(56,5) \\
64(60,4)\end{array}$ & $p=0,547^{\dagger}$ \\
\hline Idade & 230 & $69,0(65,0-77,0)$ & $70,0(65,0-77,0)$ & $p=0,863^{\ddagger}$ \\
\hline ASA $\begin{array}{r}1 \\
2 \\
3 \\
4\end{array}$ & $\begin{array}{c}10 \\
125 \\
85 \\
10\end{array}$ & $\begin{array}{c}6(60,0) \\
56(44,8) \\
32(37,6) \\
2(20,0)\end{array}$ & $\begin{array}{c}4(40,0) \\
69(55,2) \\
53(62,4) \\
8(80,0)\end{array}$ & $p=0,046^{\pi}$ \\
\hline Score Charlson & 230 & $5,0(3,0-9,0)$ & $7,0(4,0-10,0)$ & $p=0,017^{\neq}$ \\
\hline MoCA & 230 & $22,0(18,0-26,0)$ & $22,0(18,0-26,0)$ & $p=0,976^{*}$ \\
\hline $\begin{array}{l}\text { Fragilidade clínica } \\
\text { Robusto } \\
\text { Bem/saudável } \\
\text { Controlado } \\
\text { Vulnerável } \\
\text { Fragilidade leve } \\
\text { Fragilidade moderada } \\
\text { Fragilidade severa }\end{array}$ & $\begin{array}{l}24 \\
46 \\
71 \\
39 \\
16 \\
21 \\
13\end{array}$ & $\begin{array}{l}11(45,8) \\
21(45,7) \\
27(38,0) \\
14(35,9) \\
9(56,2) \\
9(42,9) \\
5(38,5)\end{array}$ & $\begin{array}{c}13(54,2) \\
25(54,3) \\
44(62,0) \\
25(64,1) \\
7(43,8) \\
12(57,1) \\
8(61,5)\end{array}$ & $p=0,844 \pi$ \\
\hline $\begin{array}{l}\text { Antecedentes de EAM } \\
\text { Sim } \\
\text { Não }\end{array}$ & $\begin{array}{c}16 \\
214\end{array}$ & $\begin{array}{c}6(37,5) \\
90(42,1)\end{array}$ & $\begin{array}{c}10(62,5) \\
124(57,9)\end{array}$ & $p=0,721^{+}$ \\
\hline $\begin{array}{l}\text { Antecedentes de AVC } \\
\text { Sim } \\
\text { Não }\end{array}$ & $\begin{array}{c}15 \\
215\end{array}$ & $\begin{array}{l}2(13,3) \\
94(43,7)\end{array}$ & $\begin{array}{c}13(86,7) \\
121(56,3)\end{array}$ & $p=0,021^{+\star}$ \\
\hline $\begin{array}{l}\text { Antecedentes de demência } \\
\text { Sim } \\
\text { Não }\end{array}$ & $\begin{array}{c}6 \\
224\end{array}$ & $\begin{array}{l}2(33,3) \\
94(42,0)\end{array}$ & $\begin{array}{c}4(66,7) \\
130(58,0)\end{array}$ & $p=0,508$ \\
\hline $\begin{array}{l}\text { Antecedentes de patologia } \\
\text { neurológica } \\
\text { Sim } \\
\text { Não }\end{array}$ & $\begin{array}{c}17 \\
213\end{array}$ & $\begin{array}{c}8(47,1) \\
88(41,3)\end{array}$ & $\begin{array}{c}9(52,9) \\
125(58,7)\end{array}$ & $p=0,644^{+}$ \\
\hline $\begin{array}{l}\text { Antecedentes de patologia } \\
\text { psiquiátrica } \\
\text { Sim } \\
\text { Não }\end{array}$ & $\begin{array}{c}33 \\
197\end{array}$ & $\begin{array}{l}15(45,5) \\
81(41,1)\end{array}$ & $\begin{array}{c}18(54,5) \\
116(58,9)\end{array}$ & $p=0,640^{+}$ \\
\hline $\begin{array}{l}\text { Antecedentes de anemia } \\
\text { Sim } \\
\text { Não }\end{array}$ & $\begin{array}{c}47 \\
183\end{array}$ & $\begin{array}{l}13(27,7) \\
83(45,4)\end{array}$ & $\begin{array}{c}34(72,3) \\
100(54,6)\end{array}$ & $p=0,028^{+\star}$ \\
\hline $\begin{array}{l}\text { Insuficiência cardíaca } \\
\text { congestiva } \\
\text { Sim } \\
\text { Não }\end{array}$ & $\begin{array}{c}18 \\
212\end{array}$ & $\begin{array}{l}7(38,9) \\
89(42,0)\end{array}$ & $\begin{array}{c}11(61,1) \\
123(58,0)\end{array}$ & $p=0,798^{\dagger}$ \\
\hline $\begin{array}{l}\text { Doença cardíaca isquémica } \\
\text { Sim } \\
\text { Não }\end{array}$ & $\begin{array}{c}19 \\
211\end{array}$ & $\begin{array}{l}8(42,1) \\
88(41,7)\end{array}$ & $\begin{array}{c}11(57,9) \\
123(58,3)\end{array}$ & $p=0,973^{+}$ \\
\hline $\begin{array}{l}\text { DPOC } \\
\text { Sim } \\
\text { Não }\end{array}$ & $\begin{array}{c}11 \\
219\end{array}$ & $\begin{array}{c}4(36,4) \\
92(42,0)\end{array}$ & $\begin{array}{c}7(63,6) \\
127(58,0)\end{array}$ & $p=0,484^{\|}$ \\
\hline $\begin{array}{l}\text { SAOS } \\
\text { Sim } \\
\text { Não }\end{array}$ & $\begin{array}{c}10 \\
220\end{array}$ & $\begin{array}{l}4(40,0) \\
92(41,8)\end{array}$ & $\begin{array}{c}6(60,0) \\
128(58,2)\end{array}$ & $p=0,590^{\|}$ \\
\hline $\begin{array}{l}\text { Insuficiência renal crónica, } \\
\text { Sim } \\
\text { Não }\end{array}$ & $\begin{array}{c}20 \\
210\end{array}$ & $\begin{array}{l}6(30,0) \\
90(42,9)\end{array}$ & $\begin{array}{c}14(70,0) \\
120(57,1)\end{array}$ & $p=0,265^{\dagger}$ \\
\hline $\begin{array}{l}\text { Diabetes } \\
\text { Sim } \\
\text { Não }\end{array}$ & $\begin{array}{c}57 \\
173\end{array}$ & $\begin{array}{l}19(33,3) \\
77(44,5)\end{array}$ & $\begin{array}{l}38(66,7) \\
96(55,5)\end{array}$ & $p=0,138^{+}$ \\
\hline $\begin{array}{l}\text { Patologia tiroideia } \\
\text { Sim } \\
\text { Não }\end{array}$ & $\begin{array}{c}25 \\
205\end{array}$ & $\begin{array}{l}10(40,0) \\
86(42,0)\end{array}$ & $\begin{array}{c}15(60,0) \\
119(58,0)\end{array}$ & $p=0,852^{\dagger}$ \\
\hline $\begin{array}{l}\text { Técnica anestésica } \\
\text { Geral } \\
\text { Combinada } \\
\text { Regional } \\
\text { Sedação }\end{array}$ & $\begin{array}{l}137 \\
50 \\
32 \\
11\end{array}$ & $\begin{array}{l}55(40,1) \\
23(46,0) \\
13(40,6) \\
5(45,5)\end{array}$ & $\begin{array}{l}82(59,9) \\
27(54,0) \\
19(59,4) \\
6(54,5)\end{array}$ & $p=0,898^{*}$ \\
\hline
\end{tabular}

P25 - P75: intervalo entre percentis; Cl: consentimento informado; ASA: classificação de estado físico de acordo com a classificação da Sociedade Americana de Anestesiologistas; MoCA: capacidade cognitiva avaliada pelo Montreal Cognitive Assessment; EAM: enfarte agudo do miocárdio; AVC: acidente vascular cerebral; DPOC: doença pulmonar obstrutiva crónica; SAOS: síndrome da apneia obstrutiva do sono.

* Estatisticamente significativo, $p<0,05$; † Teste do $\chi^{2}$ de Pearson; * Teste de Mann-Whitney; ${ }^{\pi}$ Teste linear-by-linear association; ' Teste exato de Fisher; ${ }^{*}$ Teste qui-quadrado 
regulação são vinculativos para todos os médicos inscritos na Ordem dos Médicos e visam garantir a adequação da prática médica aos parâmetros de qualidade exigidos. A obrigatoriedade do $\mathrm{Cl}$ está preconizada no Estatuto ${ }^{7}$ (art. $135^{\circ}$ ) e no Código Deontológico da Ordem dos Médicos ${ }^{8}$ (art. $19^{\circ}$ e $20^{\circ}$ ). A responsabilidade disciplinar é invocada aquando da violação (por ação ou omissão) dos deveres e regras de conduta específicas da classe médica, cabendo exclusivamente à Ordem dos Médicos o exercício da ação disciplinar. ${ }^{42}$

Quanto à responsabilidade civil, as ações por responsabilidade médica podem ser sobretudo de dois tipos: por má prática ou erro técnico; ou por violação dos direitos dos doentes, nomeadamente os direitos à autodeterminação e liberdade do doente, intimamente relacionados com o conceito de $\mathrm{Cl}^{4}{ }^{4} \mathrm{No}$ âmbito da responsabilidade civil (contratual ou extracontratual) é necessário que exista uma conduta voluntária da qual resulte ilicitamente um dano no doente, um nexo de causalidade adequada entre o facto praticado pelo médico e o dano e, seja demonstrado, que o médico atuou com culpa. ${ }^{11}$

O Código Civil $^{9}$ visa proteger os bens jurídicos de direito à autodeterminação, integridade física e integridade moral da pessoa humana. As intervenções cirúrgicas não são isentas de riscos que podem condicionar um agravamento do estado de saúde do doente, pelo que é fundamental que este esteja esclarecido acerca dessas possíveis complicações. O consentimento esclarecido tem importância fulcral no estabelecimento da licitude da intervenção médico-cirúrgica (artigo $340^{\circ}$ do Código Civil). ${ }^{9}$ A sua ausência pode estar implicada em processos litigiosos por danos não patrimoniais (por violação do direito à autodeterminação e liberdade) ou patrimoniais (por agravamento do estado de saúde no contexto de intervenções médico-cirúrgicas arbitrárias). O médico terá, então, o dever de indemnizar o doente pelos danos sofridos com a sua atuação. ${ }^{43}$

De acordo com o artigo $150^{\circ}$ do Código Penal, ${ }^{10}$ as intervenções médico-cirúrgicas realizadas por médicos ou outros profissionais devidamente qualificados, com finalidade terapêutica e de acordo com as leges artis não são consideradas ofensas à integridade física/corporal. Se, por sua vez, uma intervenção for realizada de acordo com as leges artis, de forma tecnicamente correta, porém sem consentimento adequado (e válido de acordo com os pressupostos do artigo $157^{\circ}$ ), constitui uma intervenção médico-cirúrgica arbitrária e crime contra a liberdade pessoal, punível até três anos de prisão ou com pena de multa. Quando o médico atua possibilitando um risco não consentido pelo doente e este se concretiza, pode enfrentar processo penal por ofensa à integridade física por negligência (art. 148 ${ }^{\circ}$ ) ou homicídio por negligência (art. 137 $) .44 \mathrm{O}$ consentimento permite apenas afastar violações da autodeterminação do doente, não de outros bens jurídicos. ${ }^{45}$

Apesar da importância do formulário de $\mathrm{Cl}$, são conhecidas decisões de tribunais estrangeiros, nomeadamente o australiano e o britânico, ${ }^{46,47}$ que recusaram o valor destes documentos com o argumento de que haviam sido reduzidos a formalidades desprovidas de informação apropriada (em alguns estudos, menos de metade dos doentes foram informados acerca das alternativas terapêuticas) ${ }^{48}$ ou compreensão pelo doente da situação a consentir (uma percentagem significativa de doentes assina o consentimento sem ter compreendido o seu direito legal ao mesmo). ${ }^{48} \mathrm{Em}$ Portugal, existe ainda pouco contacto com processos de responsabilidade médica relacionados com o $\mathrm{Cl}$ (Acórdãos de 09/10/2014 e 7/03/2017 do Supremo Tribunal de Justiça), ${ }^{49,50}$ e nenhum processo por ausência de $\mathrm{Cl}$ por escrito para o ato anestésico.

A mera assinatura de um formulário não é, portanto, suficiente para garantir a validade do consentimento enquanto vontade devidamente esclarecida e livre, algo que está claramente estabelecido na Norma 015/2013 da Direção-Geral da Saúde, atualizada a 04/11/20153: "o processo de obtenção do $\mathrm{Cl}$ não se esgota no procedimento administrativo de obter a anuência escrita ou verbal do doente para o ato proposto, devendo ser uma atitude sistemática e contínua do exercício dos profissionais de saúde".

Muitos doentes desconhecem termos médicos e têm noções erradas acerca do procedimento anestésico, ${ }^{13,51}$ que devem ser esclarecidas. A prática do $\mathrm{Cl}$ para o ato anestésico melhora a compreensão acerca dos procedimentos anestésicos, seus benefícios e efeitos laterais por parte dos doentes. ${ }^{13,14}$ Apesar da inexistência de um consenso acerca da informação a discutir sistematicamente com os doentes,

Tabela 2 - Distribuição dos doentes observados por serviço cirúrgico $(n=230)$

\begin{tabular}{lccc}
$\begin{array}{l}\text { Serviço } \\
\mathrm{n}(\%)\end{array}$ & $\begin{array}{c}\text { Total } \\
\mathrm{n}=230\end{array}$ & $\begin{array}{c}\text { Com Cl por escrito } \\
\text { para ato anestésico } \\
\mathrm{n}=96(41,7)\end{array}$ & $\begin{array}{c}\text { Sem Cl por escrito } \\
\text { para ato anestésico } \\
\mathrm{n}=134(58,3)\end{array}$ \\
\hline Cirurgia Geral & 92 & $37(40,2)$ & $55(59,8)$ \\
Cirurgia Vascular & 21 & $8(38,1)$ & $13(61,9)$ \\
Ginecologia & 11 & $6(54,5)$ & $5(45,5)$ \\
Ortopedia & 32 & $18(56,3)$ & $14(43,8)$ \\
Cirurgia Plástica & 14 & $5(35,7)$ & $9(64,3)$ \\
Urologia & 53 & $19(35,8)$ & $34(64,2)$ \\
Cirurgia Maxilo-Facial & 7 & $3(42,9)$ & $4(57,1)$ \\
Total, $\mathrm{n}(\%)$ & 230 & $96(41,7)$ & $134(58,3)$ \\
\hline
\end{tabular}

Teste exato de Fisher; Cl: consentimento informado 
é sempre sensato discutir: qual o procedimento proposto e porquê; benefícios e resultados esperados; riscos, complicações e consequências; alternativas à proposta. O critério de adequação e suficiência da informação partilhada para fins de autodeterminação passa pelo padrão do doente concreto. ${ }^{42}$ Deve procurar-se transformar a obrigação legal em incentivo ao diálogo com troca de informação adequada a cada situação particular e adequação do plano às preocupações, características individuais e probabilidade de cada outcome no doente concreto. ${ }^{38}$ Estes fatores podem influenciar a proposta anestésica, nomeadamente na decisão por anestesia geral ou regional pelas suas características e riscos distintos. ${ }^{52}$ Devem ser evitados formulários demasiado complexos ou sobrecarregados de informação, assim como linguagem demasiado técnica, que possam dificultar a compreensão pelo doente.

As falhas na informação e compreensão dos atos anestésicos podem causar insatisfação do doente ou seu representante face aos cuidados médicos e, assim, constituir fatores de risco para litígio. ${ }^{53}$ Efetivamente, a literatura existente confirma a insatisfação face à informação transmitida acerca de diferentes alternativas anestésicas, riscos e benefícios do procedimento. ${ }^{12}$ Verificou-se, também, pouca consistência entre a informação partilhada por diferentes anestesiologistas de uma mesma instituição. ${ }^{13}$ Alguns autores afirmaram já que, em determinados contextos, a inadequada comunicação entre médico e doente pode ser a principal causa de litígio, ${ }^{54}$ daí ser fundamental a avaliação pré-anestésica em consulta própria para o efeito, tal como preconizado pela norma n 029/2013 da Direção Geral de Saúde, "Avaliação Pré-Anestésica Para Procedimentos Eletivos". ${ }^{17}$

Para além de devidamente informado, o processo de $\mathrm{Cl}$ deve ser documentado adequadamente. A manutenção de registos esclarecedores, nomeadamente no processo clínico do doente, é uma prática fundamental na abordagem médica, contribuindo para o esclarecimento da validade do consentimento e prestação de cuidados médicos adequados. No entanto, outros autores verificaram que apenas uma proporção insatisfatória dos médicos o faz por rotina. ${ }^{26,55}$ Esta omissão pode dificultar o esclarecimento acerca da validade do $\mathrm{Cl}$.

Os anestesiologistas referem várias dificuldades na obtenção do $\mathrm{Cl}$ para o ato anestésico - a atual sobrecarga do sistema nacional de saúde predispõe a que muitas vezes o anestesiologista se encontre com o doente apenas minutos antes da cirurgia, sem tempo nem condições apropriadas a uma discussão esclarecedora e livre de pressões externas. Em trabalhos anteriores, uma grande parte dos anestesiologistas referiu sentir dificuldades na seleção da informação a partilhar, ${ }^{26}$ ou devido a barreiras de comunicação e escassez de tempo. ${ }^{55}$ Apesar de constituírem uma realidade, estas dificuldades não podem justificar a ausência do $\mathrm{Cl}$ enquanto imperativo ético-legal.

Neste estudo verificámos não existirem diferenças entre a obtenção de $\mathrm{Cl}$ por escrito para o ato anestésico entre as diferentes intervenções cirúrgicas, o que permite inferir que a insuficiente implementação do $\mathrm{Cl}$ para o ato anestésico é transversal a toda a instituição. Foi utilizado um formulário de $\mathrm{Cl}$ previamente criado pela Comissão de Ética Institucional. Este apresenta linguagem simples e acessível, e informação sucinta de forma a incentivar a discussão oral, sendo prático para o uso sistemático na prática clínica.

Quanto às limitações deste estudo, foi estudado apenas a população idosa e um pequeno número de doentes, um número limitado de serviços hospitalares, e somente as práticas de um único hospital.

\section{CONCLUSÃO}

Tendo em conta as limitações inerentes do presente trabalho, previamente inumeradas, foi-nos possível identificar uma diminuta implementação do $\mathrm{Cl}$ por escrito para o ato anestésico. Existe a necessidade de generalização e uniformização do processo de obtenção do $\mathrm{Cl}$ para os atos anestésicos. A avaliação pré-operatória, a prática de $\mathrm{Cl}$ específico para o ato anestésico e o seu registo são fundamentais para o esclarecimento do doente acerca do status da Anestesiologia enquanto especialidade distinta e complementar na intervenção médico-cirúrgica.

$\mathrm{O} \mathrm{Cl}$ é um dever ético, disciplinar e legal em qualquer intervenção, que visa proteger a autodeterminação do doente, reconhecido nas várias normas legais e deontológicas. A autonomia do anestesiologista e ato anestésico está consagrada e este tem, portanto, a obrigação de obter o $\mathrm{Cl}$ para esse procedimento. Para ser válido, o consentimento deve ser adequadamente informado e documentado, contribuindo para a prevenção e esclarecimento de eventual processo litigioso. A ausência de $\mathrm{Cl}$ válido constitui uma violação das boas práticas médicas e do bem jurídico de liberdade, podendo ser invocada a responsabilidade disciplinar, civil ou penal do médico responsável.

Apesar das dificuldades na sua implementação regular, a obtenção de $\mathrm{Cl}$ escrito para o ato anestésico é de grande importância e não deve ser negligenciada. Seria também pertinente estudar a prática do $\mathrm{Cl}$ por escrito numa população mais jovem, de modo a obter comparações e estudar uma possível subvalorização da discussão com doentes mais idosos e sua capacidade de autodeterminação. Seria igualmente pertinente, verificar se esta realidade é transversal a outros Centros Hospitalares Portugueses, mesmo não universitários, e quais as razões para a não implementação do consentimento anestésico de uma forma sistemática.

\section{PROTEÇÃO DE PESSOAS E ANIMAIS}

Os autores declaram que os procedimentos seguidos estavam de acordo com a Declaração de Helsínquia da Associação Médica Mundial.

\section{CONFIDENCIALIDADE DOS DADOS}

Os autores declaram ter seguido os protocolos do seu centro de trabalho acerca da publicação de dados. 


\section{CONFLITO DE INTERESSES}

Os autores declaram que não há conflito de interesses na publicação deste artigo.

\section{REFERÊNCIAS}

1. Entidade Reguladora da Saúde. Consentimento Informado - Relatório final. Maio de 2009. Lisboa: ERS; 2009.

2. Tait AR, Teig MK, Voepel-Lewis T. Informed consent for anesthesia: a review of practice and strategies for optimizing the consent process. Can J Anesth. 2014;61:832-42.

3. Direção Geral de Saúde. Consentimento informado, esclarecido e livre dado por escrito. $\mathrm{N}^{\circ}$ 015/2013, atualizado a 04/11/2015. Lisboa: DGS; 2015.

4. Pereira AG. Responsabilidade médica e consentimento informado. Ónus da prova e nexo de causalidade. Centro de Estudos Jurídicos e Judiciários da Região Administrativa Especial de Macau, República Popular da China. Macau: CEJJ; 2008.

5. Assembleia da República. Convenção de Oviedo - Convenção sobre os Direitos do Homem e da Biomedicina (Conselho da Europa 1997). Diário da Repub. 2001;1:14-36.

6. Assembleia da República. Lei de Bases da Saúde. Lei n 48/90. Diário da Republica. 1990;195:3452-9.

7. Assembleia da República. Estatuto da Ordem dos Médicos. Lei $n^{\circ}$ 117/2015. Diário da Republica. 2015;169: 6520-79.

8. Ordem dos Médicos. Código Deontológico Da Ordem Dos Médicos. Regulamento No 707/2016. Diário da Republica. 2016;139.

9. Lei $\mathrm{N}^{\circ}$ 43/2017. Código Civil Português. 73a Versão, de 2017-06-14.; 2017.

10. Lei № 94/2017. Código Penal Português. 45ª Versão, de 2017-08-23.; 2017.

11. Nunes JM. Da responsabilidade dos médicos anestesiologistas dos diversos tipos de responsabilidade, formas de apuramento e instâncias decisórias. Consentimento informado. Coimbra: Serviço de Anestesiologia, Hospitais da Universidade de Coimbra; 2006.

12. Tait AR, Voepel-Lewis T, Gauger V. Parental recall of anesthesia information: Informing the practice of informed consent. Anesth Analg. 2011;112:918-23.

13. Zarnegar R, Brown MR, Henley M, Tidman V, Pathmanathan A. Patient perceptions and recall of consent for regional anaesthesia compared with consent for surgery. J R Soc Med. 2015;108:451-6.

14. Rampersad K, Chen D, Hariharan S. Efficacy of a separate informed consent for anesthesia services: A prospective study from the Caribbean. J Anaesthesiol Clin Pharmacol. 2016;32:18-24.

15. Klock PA, Roizen MF. More or better - educating the patient about the anesthesiologist's role as perioperative physician. Anesth Analg. 1996;83:671-2.

16. Marco AP. Informed consent for surgical anesthesia care: Has the time come for separate consent? Anesth Analg. 2010;110:280-2.

17. Direção Geral de Saúde. Avaliação pré-anestésica para procedimentos eletivos. № 029/2013, atualizado a 24/04/2015. Lisboa: DGS; 2015.

18. Quan H, Li B, Couris CM, Fushimi K, Graham P, Hider P, et al. Updating and validating the charlson comorbidity index and score for risk adjustment in hospital discharge abstracts using data from 6 countries. Am J Epidemiol. 2011;173:676-82.

19. Charlson ME, Pompei P, Ales KL, MacKenzie CR. A new method of classifying prognostic comorbidity in longitudinal studies: development and validation. J Chronic Dis. 1987;40:373-83.

20. Rockwood K, Song X, MacKnight C, Bergman H, Hogan DB, McDowell I, et al. A global clinical measure of fitness and frailty in elderly people. CMAJ. 2005;173:489-95.

21. Islam A, Muir-Hunter SW, Speechley M, Montero-Odasso M. Facilitating frailty identification: comparison of two methods among communitydwelling order adults. J Frailty Aging. 2014;3:216-21.

22. Freitas S, Simões MR, Marôco J, Alves L, Santana I. Construct validity of the Montreal Cognitive Assessment (MoCA). J Int Neuropsychol Soc. 2012;18:242-50.

23. Freitas S, Simões MR, Martins C, Vilar M, Santana I. Estudos de adaptação do Montreal Cognitive Assessment (MOCA) para a população portuguesa. Avaliação Psicol. 2010;9:345-57.

24. Nasreddine ZS, Phillips NA, Bédirian V, Charbonneau S, Whitehead V, Collin I, et al. The Montreal Cognitive Assessment, MoCA: a brief screening tool for mild cognitive impairment. J Am Geriatr Soc. 2005;53:695-9.

\section{FONTES DE FINANCIAMENTO}

Os autores declaram não ter recebido subsídios ou bolsas para a elaboração do artigo.

25. Lichtenberg PA. Handbook of assessment in clinical gerontology. . $2^{\text {nd }}$ ed. Philadelphia: Academic Press; 2010.

26. Momin SG. Survey of anaesthesiologists' reactions to implementation of laws related to anaesthesia practice. Indian J Anaesth. 2015;59:103-9.

27. Fleisher LA, Roizen MF. Essence of anesthesia practice. $3^{\text {rd }}$ ed. Philadelphia: Elsevier/Saunders; 2011.

28. Teles $R$, Mansilha A. Vantagens da anestesia locoregional relativamente à anestesia geral na endarterectomia carotídea. Angiol Cir Vasc. 2014;10:64-70.

29. Zugliani A. Anestesia em ortopedia e bloqueio de nervos periféricos. Philadelphia: Elsevier/Saunders; 2016.

30. Karkouti K, Wijeysundera DN, Beattie WS. Risk associated with preoperative anemia in cardiac surgery: a multicenter cohort study. Circulation. 2008;117:478-84.

31. Musallam KM, Tamim HM, Richards T, Spahn DR, Rosendaal FR, Habbal A, et al. Preoperative anaemia and postoperative outcomes in noncardiac surgery: a retrospective cohort study. Lancet. 2011;378:1396407.

32. Hans G, Jones N. Preoperative anaemia. Contin Educ Anaesth Critical Care Pain. 2013;13:71-4.

33. Priya Khanna M, Hébert PC, Fergusson DA. Review of the clinical practice literature on patient characteristics associated with perioperative allogeneic red blood cell transfusion. Transfus Med Rev. 2003;17:110-9.

34. Hare GM, Baker JE, Pavenski K. Assessment and treatment of preoperative anemia: Continuing Professional Development. Can J Anesth. 2011;58:569-81.

35. Klick JC, Avery EG. Anesthetic considerations for the patient with anemia and coagulation disorders. In: Longnecker DE, Brown DL, Newman MF, Zapol WM, editors. Anesthesiology. $2^{\text {nd }}$ ed. Philadelphia: McGraw-Hill Education; 2012.

36. Segura C, Vidas S. Orientações da OMS para a cirurgia segura 2009 Lisboa: DGS; 2009.

37. Waisel DB, Truog RD. The benefits of the explanation of the risks of anesthesia in the day surgery patient. J Clin Anesth. 1995;7:200-4

38. Minossi JG. Prevenção de conflitos médico-legais no exercício da medicina. Rev Col Bras Cir. 2009;36:90-5.

39. Dias AS. Responsabilidade penal negligente em organizações complexas de cuidados de saúde. Responsabilidade Médica, Colóquio Da Sociedade de Advogados Morais Leitão, Galvão Teles, Soares Da Silva. 2012:52-57

40. Estatuto Disciplinar da Ordem dos Médicos. Regulamento n 631/2016 Diário Republica. 2016:130;20968-75.

41. Pereira AD. Responsabilidade Disciplinar Médica - Manual do Módulo. Curso de Responsabilidade Médica. Lisboa: Ordem dos Advogados; 2018.

42. Pereira AD. Pressupostos da Responsabilidade Civil Médica - Manual do Módulo. Curso de Responsabilidade Médica. Lisboa: Ordem dos Advogados; 2018.

43. Pereira AD. Responsabilidade Penal Médica - Manual do Módulo. Curso de Responsabilidade Médica. Lisboa: Ordem dos Advogados; 2018.

44. Raposo VL. Do regime das intervenções médico-cirúrgicas arbitrárias no código penal Português. Rev Peru Ciencias Penal. 2013:1-22.

45. High Court of Australia. Rogers v. Whitaker. Aust Law J. 1993:47-55.

46. House of Lords of The United Kingdom. Chester v Afshar. London: HL; 2004.

47. Falagas ME, Akrivos PD, VAlexiou VG, Saridakis V, Moutos T, Peppas $G$, et al. Patients' perception of quality of pre-operative informed consent in Athens, Greece: a pilot study. PLoS One. 2009;4:e8073.

48. Supremo Tribunal de Justiça. Processo 6669/11.3TBVNG.S1. Acórdão de 07-03-2017. Lisboa: STJ; 2017.

49. Supremo Tribunal de Justiça. Processo 3925/07.9TVPRT.P1.S1. Acórdão de 09-10-2014. Lisboa: STJ; 2014.

50. Hume MA, Kennedy B, Asbury AJ. Patient knowledge of anaesthesia and peri-operative care. Anaesthesia. 1994;49:715-8.

51. Green DS, MacKenzie CR. Nuances of informed consent: the paradigm of regional anesthesia. HSS J. 2007;3:115-8.

52. Stelfox HT, Gandhi TK, Orav EJ, Gustafson ML. The relation of patient satisfaction with complaints against physicians and malpractice lawsuits. 
53. Am J Med. 2005;118:1126-33.

53. Soin B, Thomson HJ, Smellie WA. Informed consent: a case for more education of the surgical team. Ann R Coll Surg Engl. 1993;75:62-5.

54. Houghton D, Williams S, Bennett J, Back G, Jones A. Informed consent: patients' and junior doctors' perceptions of the consent procedure. Clin
Otolaryngol Allied Sci. 1997:22:515-8.

55. Waisel DB, Lamiani G, Sandrock NJ, Pascucci R, Truog RD, Meyer EC. Anesthesiology trainees face ethical, practical, and relational challenges in obtaining informed consent. Anesthesiology. 2009;110:480-6. 